\title{
A Critical Analysis of HPV Vaccination in Children
}

\author{
Bittmann S*, Luchter E, Weissenstein A and Villalon G \\ Department of Pediatrics, Ped Mind Institute, Germany \\ *Corresponding author: Stefan Bittmann, Head of Department of Pediatrics, Germany. \\ To Cite This Article: Stefan Bittmann. A Critical Analysis of HPV Vaccination in Children. Am J Biomed Sci \& Res. 2019 - 4(5). AJBSR. \\ MS.ID.000822. DOI: 10.34297/AJBSR.2019.04.000822
}

Received: July 30, 2019 | Published: August 14, 2019

\section{Introduction}

Worldwide, infection with HPV viruses is the most common sexually transmitted disease. The prevalence of HPV infection varies considerably from country to country and can change significantly with changes in lifestyle and sexual behavior. Every year, about half a million women worldwide develop cervical cancer, with up to four-fifths of them from developing countries. The lack or underutilization of cancer screening programmers in developing countries plays a decisive role in this. It is estimated that around 230,000 women worldwide die of cervical cancer every year [1].

In Central Europe, cervical cancer is the eleventh most common cancer among women of all age groups. It is the second most common malignant tumor in women under 45 years of age, but accounts for only $3.2 \%$ of all cancers and $1.8 \%$ of all cancer deaths in women. A multifactorial cause is propagated, whereby primarily proteins and genome parts of different HPV viruses but also other cofactors such as a young age in initial diagnosis, immunosuppression, smoking and co-infections with herpes simplex virus and infections with chlamydia play a not insignificant role. The long latency between HPV primary infection and cancer suggests that the other factors such as sexual behavior, genetic predisposition, nutritional status and social educational status may have an influence.

HPV vaccines are vaccines designed to protect against certain types of sexually transmitted human papillomavirus (HPV) and to prevent cancer. A bi- and a tetravalent vaccine are currently available. Two HPV vaccines are currently approved: Cervarix and Gardasil. Cervarix is a bivalent vaccine and only effective against HPV types 16 and 18. Gardasil is a tetravalent vaccine targeted against HPV types 6,11,16 and 18 . However, there are significantly more HPV viruses with oncogenic potential that do not occur in either of these vaccines. Regular screening for early detection of cervical cancer (PAP test) is recommended because not all carcinogenic HPV types are covered by the vaccine.

The vaccines only have a preventive and not a therapeutic effect. The aim of the vaccine is to reduce the burden of cervical cancer. If one considers the seroconversion rate of both vaccines one month after the third administration, figures of $99.5 \%$ are given, with the duration of protection 6 years [2]. In spring 2007, STIKO issued a recommendation for the vaccination of girls aged 12-17 against carcinogenic HPV types 16 and 18. In June 2007, the Joint Federal Committee (GBA) introduced vaccination as the standard benefit of the statutory health insurance fund. Ongoing intensive and well-founded discussions on the legitimacy, benefits and cost-effectiveness of HPV vaccination are the order of the day and justify a statement.

\section{Etiology and Pathogenesis of Human Papilloma Virus} Infection

The papillomavirus is a virus particle about $55 \mathrm{~nm}$ in size that includes a double-stranded circular viral genome of 7904 base pairs. HP viruses belong to the Papovavirus family and have an icosahedral protein capsid. There are approximately 100 different HPV types that can infect both the skin and mucous membranes. In the area of the uro-anogenital tract about 40 different HPV types come to the fore, which are divided into 2 groups. The lowrisk HPV types have a low potential to cause a malignant disease. High-risk HPV types have a high oncogenic potential. In Europe, the infection rate without clinically relevant change is between 1633 million people/year. Infection with human papilloma viruses is one of the most common sexually transmitted diseases worldwide. Approximately $70 \%$ of all people have been infected with genital HPV at least once during their lifetime.

Women generally become infected between the ages of 20 and 30 , with one in three women showing HPV two years after their first sexual contact [3]. HPV causes persistent infections of the uterus with an increased risk of developing precancerous precursors (CIN). In general, the time span between primary infection and cancer development can be up to 20 years or longer, whereby the incidence of cervical cancer is significantly increased from the age of 35 [4]. However, in about $10 \%$ of chronically infected individuals, the changes progress to high-grade dysplasia (CIN III) within eight 
years, which is considered a precursor of cancer. These changes also recede by up to 3\%, with approximately 8-12 years between CIN III and cancer [5].

\section{Molecular Biology and Oncogenesis of HPV Infection}

The hullless virus has a simple structure and is divided into an early region, which performs regulatory functions, and a late region, which encodes two structural proteins. The capsid of the virus consists of $80 \%$ structural protein L1 and 20\% capsid protein L2. In the "early region" there are 6 viral genes, 2 of which are called oncogenes. They are responsible for virus replication and the transformation of infected cells. Papillomaviruses infect the basal cell layer of the epithelium and initially lead to a latent infection, in which the viral genome are propagated in parallel with the host cell. Clinically, these infections remain inapparent. In some cells, replication of papillomaviruses occurs and finally the release of viral capsids in the area of the epithelial layers with abraded epithelial remains.

This stage of cell change with pronounced virus proliferation corresponds in histological section to a mild cervical lesion (CIN 1) with detection of coilocytes corresponding to a vesicular distension of the cells. However, if the oncogenic oncogenes are expressed in cells replicating their own genome, malignant transformation may occur. Dysplastic cells develop, which can develop into highly dysplastic lesions. Unregulated and increased cell proliferation sets in, which ultimately leads to cancer growth. In addition, a further cell protein, p16INK4a, is produced by this process, which would prevent the cell from dividing again during normal cell division through strict feedback. The detection of this protein indicates advanced HPV infection and is a surrogate marker for the activated oncogene expression of HR-HP viruses in dysplastic cervical epithelial cells. In cervical cancer, genetic HPV can be detected in $95 \%$ of tissue samples, suggesting a causal relationship. However, there are also forms of cervical cancer without the presence of HPV hereditary material.

\section{Risks of HPV Infection}

High-risk HPV types 16 and 18 are considered responsible for about $70 \%$ of all cervical cancers in women worldwide [6]. 55.3\% of all cervical precancerous lesions of severity CIN 2 and 3 were associated with HPV 16 in 2 studies, 6.4\% with HPV 18, HPV 45 in $8.5 \%$ and HPV 31 in $6.4 \%$ of cases [7]. In 2005 the WHO classified the HPV types 16,18,31,33,35,39,45,51,56,58,59 and 66 as carcinogenic [8]. the so-called low-risk HPV types 6 and 11 are responsible for the development of over $90 \%$ of genital warts. Genital warts are the most common viral sexually transmitted disease worldwide.

\section{Frequency, Age Distribution and Course of Cervical} Cancer

In Germany, about 6200 women develop cervical cancer. With 1700 deaths, cervical carcinoma was not one of the most common cancers with $1.8 \%$ of all cancer deaths and not one of the most frequent causes of cancer deaths in women. In the incidence statistics it is currently only in 11th place. This actual state is the result of an effective early detection programme. The comparatively low incidence of cervical cancer is accompanied by a much higher incidence of found dysplasias. The EUROCARE-4 study published in September 2007 calculated a relative 5-year survival rate for invasive cervical cancer.

\section{Early Detection Possibilities-Benefits and Risks}

Since the introduction of the cytological examination of cervical smears for the early detection of cervical cancer and its precursors (Pap test) at the age of 20 years at the beginning of the 1970s, the incidence of invasive carcinomas in Germany has decreased by about two thirds. The Pap test is a very successful and effective secondary preventive measure against cervical carcinoma. In countries such as England, Sweden and the Netherlands, screening is much better organised and therefore more effective, with a $90 \%$ reduction in the risk of cervical cancer among participants [9]. In countries without appropriate cancer screening programs, cervical cancer contributes significantly to cancer mortality.

\section{Development Aspects of HPV Prophylactic Vaccines}

A quadriple vaccine manufactured by Sanofi Pasteur MSD (Whitehouse Station, NJ) was first approved in the United States in 2006 and by the European Medicines Agency in the European Union in September 2006 through the centralized approval process. In Europe, the vaccine is marketed under the trade names Gardasil and Silgard. The vaccine contains purified, recombinantly produced L1 proteins from the capsid of the four papillomavirus types 6, 11, 16 and 18, which spontaneously combine to form viruslike particles (VLP) [10]. These L1 proteins, empty virus particles that do not contain any genetic material but look like real viruses to the immune system, are the decisive building block of the HPV vaccine. These L1-VLPs induce an amazingly high immune response during intramuscular injection.

The publication of the first HPV vaccination study in the New England Journal of Medicine in November 2002 caused a sensation due to its resounding success and was euphorically commented with the title "The beginning of the end of cervical cancer? According to the European Medicines Agency, 1.5 million patients had already been vaccinated with Gardasil in Europe by January 2008 [11]. Since October 2009, Gardasil has also been approved in the USA for the prevention of genital warts in men and boys $[12,13]$. Approval for the bivalent vaccine developed by Glaxo Smith Kline (Rixenarts, Belgium) and marketed under the trade name Cervarix was granted in Australia in May 2007 and in the European Union in September 2007. Cervarix also contains recombinant L1 proteins from the capsid in VLPs, but only papilloma virus types 16 and 18 [14]. Merck \& Co and Glaxo Smith Kline have granted each other cross-licenses allowing both to use the patent rights for vaccine 
production. The German Cancer Research Center is a co-patent holder and thus shares in the profits from the sale of the vaccine.

\section{Efficacy Aspects of The Tetravalent Vaccine (Gardasil)}

The efficacy of the tetravalent vaccine was investigated in four placebo-controlled, randomized and double-blind Phase II and Phase III studies. In women who were not infected with the corresponding human papilloma virus at the time of vaccination, the vaccine prevented infection in $96-100 \%$ of cases. In the (socalled) Future II study, the occurrence of CIN II or more severe precancerous lesions of the cervix was recorded. While a case of CIN occurred in the group of vaccinated women ( $n=5305), 42$ cases occurred in the group of women vaccinated with placebo $(n=5260)$.

For ethical reasons, the independent Data Safety Monitoring Board recommended rapid vaccination of placebo vaccinated subjects [15,16]. Including women with HPV types 6,11,16 and/ or 18 infections at baseline and those who received less than three required doses of vaccine, the efficacy of Gardasil is lower, but still present, compared to precancerous cervical cancer precursors caused by the corresponding HPV types. In the combined interim analysis of the four relevant efficacy studies conducted for approval, the efficacy of Gardasil was only 39\% [10]. Gardasil is known to cross-protect with the phylogenetically related HPV types 45, 52 and 58 [17-19].

\section{Efficacy Aspects of The Bivalent Vaccine (Cervarix)}

Cervarix is a bivalent vaccine effective against HPV 16 and 18. Statistically significant efficacy has been demonstrated for this drug in a large study only for HPV 16 and not for HPV 18. Clinical data for Cervarix are only available for a period of 5.5 years [20]. New data suggest that Cervarix may also protect against infections with HPV types not included in the vaccine. This cross-protection extends to varying degrees to virus types 31,33,35,39,45,51,52,56,58 and 59. Virus types 31 and 45 have a high oncogenic potential. According to data from the PATRICIA study (Papilloma Trial against Cancer in young Adults), a Phase III study with Cervarix involving 18,644 women aged 15-25 years in 14 countries in North and South America, Europe and the Asia-Pacific region, a reduction in CIN 2 findings of $70 \%$ (33 compared to 110 cases) and CIN 3 findings of $87 \%$ was found.

\section{Up-To-Date Data on Security and Effectiveness}

According to the data available so far, a follow-up of up to 6.4 years for women who were not infected with the highly carcinogenic HPV types 16 and 18 at the time of vaccination, almost $100 \%$ protection against cervical dysplasias induced by these two HPV serotypes was found. This fact is supported by a current German HPV vaccination guideline and a systematic review of 6 randomized trials with over 40000 women and girls aged 15 to 26 years to investigate the efficacy of the vaccines confirmed [21-23]. About the effectiveness of the vaccination against HPV 16 and 18, the study situation is increasingly consolidating. Looking at all randomized studies carried out so far, the mean observation period was 3 years, in one study 5.5 years [24] and 6.4 years [21] respectively. During this period, the vaccination fully met expectations. This is confirmed by the systematic review of Rambout [23].

In women who were tested negative for HPV 16 and 18 before vaccination and received all 3 vaccine doses, a higher degree of cell change (CIN II and higher) occurred in one of 8301 cases, in the placebo group in 86 of 8268 cases, a reduction from about $1 \%$ to $0.01 \%$. The two vaccines do not influence the course of HPV infection and therefore have no therapeutic effect. Virus elimination is not accelerated [25]. New possibilities for post-infection prophylaxis are being sought. Whether the vaccination protects against an invasive cervical carcinoma is a question that is currently in doubt. So far, only a reduction of the obligatory precancerous stages has been proven in studies. It is completely unclear how long the vaccination will last. Evidence of protection lasting beyond 5 years was provided by results after renewed administration of Gardasil after 5 years; this "antigen challenge" led to a renewed increase in antibody titres within one week.

\section{Boys and HPV Vaccination}

Boys aged 9-15 years developed immunity after vaccination. The quadrivalent vaccine was judged effective in a study of 4065 boys and men aged 16-26 years [26]. The aim is to provide a protective vaccine to prevent anal and penile cancer, which is also associated with HPV infections.

\section{Vaccination Research}

Various key questions are at stake in the research of new vaccines against HPV: There are more than 100 different types of HPV. Which HPV types have clear oncogenic potential in the development of cervical cancer has not yet been fully clarified. An oncogenic potential is known in about 15 HPV types. A crossprotection is discussed. The prevalence of HPV infections and the spread of different HPV types varies considerably geographically [27]. HPV 16 types and HPV 18 types are most frequently detected, with $85 \%$ being found in women with adenocarcinoma [28]. In a quarter of invasive cervical cancer cases, 16 HPV types other than 16 and 18 are detected.

The distribution of these 16 HPV types shows a considerable geographical variation. If critical epidemiological data are analyzed, a bivalent HPV vaccine with HPV 16 and 18 in Europe protects 71\% against cervical cancer; however, depending on the geographical location, this protection may be higher or lower (74\% in Asia, 64\% in parts of Africa) [29]. Ultimately, the goal should be to develop a multivalent vaccine that includes all HPV viruses with oncogenic potential. For example, a vaccine containing the most common 7 oncogenic HPV viruses 16,18,45,31,33,52 and 58 would provide up to $85-91 \%$ cervical cancer protection (depending on geographical 
location) [29]. Although technically feasible, the production of a multivalent HPV vaccine is currently extremely difficult, not to mention the significant production costs.

\section{Cost Aspects of HPV Vaccination}

Some studies conclude that HPV vaccination is cost-effective $[17,30,31]$. In Germany, a single injection costs approximately 150 euros, with 3 vaccinations required per patient. The basic immunisation with three injections in the months 0,2 and 6 costs about 450 Euro. According to estimates by the Federal Association of Company Health Insurance Funds, this burdens the German health system with up to one billion euros a year. Should booster vaccinations prove necessary every 5 years or so, the cost of the vaccination program would multiply. In other countries the vaccine is considerably cheaper (e.g. Australia 96 Euro). In the USA, for example, vaccinating women aged 35 and over is not cost-effective [32].

Since 2002, the pharmaceutical "vaccine industry" has experienced rapid growth. In 2002, the turnover of pharmaceutical companies in the USA from HPV vaccination was 135 million dollars, in 2012 it was already 1.4 trillion dollars [33]. Even Nobel Prize winner Professor Harald zur Hausen [34] has pointed out at every opportunity that the vaccine is too expensive. This is particularly true in developing countries, where cervical cancer is partly the most common cancer in women [30]. In Kenya, the vaccine costs about half an average annual income $[35,36]$. In developing countries, where cervical cancer is by far the most prevalent, vaccination is an almost unrealizable cost factor for the population.

\section{Adverse Side Effects of HPV Vaccination}

Data on the tolerability of the HPV vaccine were available from several clinical studies with more than 20000 participants at the time of approval. Since the approval of Gardasil, several million girls and women have been vaccinated worldwide. Unwanted side effects are registered in special monitoring programs. Based on the available study data, the HPV vaccination is assessed as safe and well tolerated by the German Health Technology Assessment, the American Drug Administration (FDA), the Centers for Disease Control and Prevention (CDC) and the European Medicines Agency $[17,37]$.

The most common adverse events in the controlled trials were local reactions in both vaccines in $83 \%$ of women in the vaccine group and in $73 \%$ of women in the placebo group. The most common systemic responses were headache, fatigue, muscle aches and nausea, which occurred equally in the vaccine and placebo groups. Severe adverse effects were bronchospasm, hypertension and severe headache. Since the US approval of the HPV vaccine Gardasil [38], the FDA and the Centers for Disease Control and Prevention have received-17160 reports-on potential adverse events from a total of approximately 26 million doses of vaccine administered as part of monitoring programmes up to September
2009. Most adverse events, $92 \%$, were not considered serious $8 \%$ were serious.

Severe was defined as a condition that leads to hospital admission, a life-threatening condition, or a serious illness, an irreversible disability or death. For example, cases of GuillainBarre syndrome (the synonym "Gardasil-Guillain Barre syndrome" is frequently used due to its frequency), thromboses and deaths have been reported in close temporal connection with vaccination. Unexpectedly, no causal link between the vaccination and the disease was found [37]. The US consumer protection group "Judical Watch" had already published a first list with 1637 reported side effects after HPV vaccination in the USA in May 2006. In October 2007, they announced a further 1824 vaccine adverse events. This resulted in 3461 adverse events from May 2006 to October 2007, including 11 deaths caused solely by HPV vaccination. On 20 June 2007, a healthy 17-year-old woman was vaccinated with Gardasil for the first time and died on the same day. Further isolated deaths have occurred and are known. Causal connections with an HPV vaccination were always denied.

\section{Demand for Re-Evaluation of HPV Vaccination}

In November 2008, scientists from various German research institutions called for the HPV vaccine to be re-evaluated on the grounds that its efficacy could be significantly lower than assumed $[39,40]$. In a re-evaluation of the Robert Koch [41] Institute on 10 August 2009, the vaccination was still recommended. However, information media that give the impression that HPV vaccination provides $100 \%$ protection against cervical cancer were currently considered to be dubious [41]. In Germany, the initial euphoria of HPV vaccination has quickly subsided. At present, the vaccination rate should be below 30\%. In June 2011, vaccine manufacturers cheered for an Australian study showing a significant decrease in cervical cell changes in HPV-vaccinated 17-year-old girls [42].

However, cheers drowned out that this effect was not detectable in the 18-year-old girls who had also been vaccinated [42]. In addition, the current data do not show any efficacy in girls and women already infected with HPV. One study even suspected that vaccination could promote the development of cell dysplasia in women already infected with HPV types 16 and 18 [38]. Therefore, vaccine manufacturers and authorities recommend vaccination only before first sexual contact. In France, the French Ministry of Health recommended that virginity be checked before vaccination. Clinical efficacy studies with girls before and during sexual maturity have not yet been carried out - manufacturers are content with the less meaningful detection of antibodies in the blood in the months following vaccination. The STIKO recommendation to vaccinate all girls before their first sexual contact is therefore on a shaky footing and is not evidence-based. These data should be available if an insufficiently studied vaccine is publicly recommended for half of the adolescent population. 


\section{Conflicts of Interest}

The European Cervical Cancer Association (ECCA) is funded by more than 50 percent by vaccine manufacturers Sanofi Pasteur, Roche, and Glaxo Smith Kline. The German Cancer Research Centre is co-owner of the cervical vaccine patents. This means that virtually every instance/person who has approved or recommended the vaccination can derive financial benefit from each individual vaccination.

\section{Conclusion}

HPV vaccination must be critically evaluated for various reasons. The approval of Gardasil was based in advance on 4 studies, for Cervarix [14] only on one phase III study. This was not enough for a meaningful assessment of the clinical benefit [43]. The clear evidence of protection against cervical cancer is not conclusive, only in cervical cancer precursors. The regulatory authorities have accepted CIN II, CIN III and Carcinoma in situ as surrogate parameters when investigating the efficacy of the potential cancer precursors. Vaccination is currently being carried out with vaccines whose clear efficacy against cervical cancer is not sufficiently known. There are no long-term studies. It is assumed that the development of cervical cancer takes 15-30 years [44]. Studies from currently only up to 6.5 years are not able to show any clarity. The protection is very often proven and determined by the level of the antibody titre; whether the level or the presence of high antibodies against HPV protects absolute protection against the development of cervical cancer is rather questionable.

In summary, it can be said: There is not yet enough knowledge available on long-term side effect risks. Cervical cancer can be adequately controlled by safer sex (good protection against risk HPV) and effective screening programmes. Its incidence has been declining for years. The vaccine is genetically engineered. What consequences it has for vaccinated people is unclear. Genetically engineered vaccines penetrate the cell material and alter it. What consequences this has for our offspring is unclear. So far, no direct causal connection has been shown that would prove that the cause of cervical carcinomas is HP viruses. Even the NCI, the National Cancer Research Institute in the USA, admits this. It is only known that factors such as the long-term use of oral contraceptives and the number of births, as well as genetic changes, smoking or an acquired immunodeficiency promotes tumor development [45-47].

It is unknown how long the vaccination protection lasts with both current vaccine alternatives. No study provides evidence for lifelong immunity; one study proves protection for at least 5 years. In this context, the question of a "booster vaccination" will become an issue. It is unknown how the vaccine will be effective. No study provides evidence of lifelong immunity; one study proves protection for at least 5 years. In this context, the question of a "booster vaccination" will become an issue. It is unknown how the vaccination affects the distribution of the other HPV types. It is unknown whether HPV 16 and 18 are increasingly replaced by other HPV types that can also have a carcinogenic effect. The vaccination creates a "sham safety" because it reduces the motivation for the early detection examination and for limiting further risks (e.g. smoking).

It is not known how the vaccination works if an infection with HPV has already occurred. It is possible that the vaccination has aggravating effects if the virus infection has already occurred but is still without symptoms. The vaccination may change the character of the other virus types not reached by the vaccination. The consequence of this situation is unknown. It is not known whether the result will be an advantage or an increase in risk. HPV vaccines are extremely expensive. Extending the early detection programme without vaccination would save high costs for the health care system, which could be invested elsewhere in social projects. Health policy is not always rational. An objective, wellfounded and balanced information of the population about benefits and limitations of HPV vaccination is desirable but probably not feasible.

\section{References}

1. Ferlay J, Bray F, Pisani P, Parkin DM (2004) International Agency for Research of Cancer (IARC) GLOBOCAN 2002: Cancer Incidence, Mortality and Prevalence Worldwide. IARC Press, France, Cancer Base p. 5.

2. De S, Kanagasabai S (2010) Human Papilloma Virus Vaccine-An Update. European J Scient Res 43(2): 256-264.

3. Franco EL, Harper DM (2005) Vaccination against human papillomavirus infection: a new paradigm in cervical cancer control. Vaccine 23(1718): 2388-2394.

4. Parkin DM, Whelan SL, Ferlay J, Storm H (2005b) Cancer Incidence in five continents. International Agency for Research on Cancer (IARC), France, Cancer Base 1(8): 7.

5. Kind E, Kuhlmann M (2004) Zervikale intraepitheliale Neoplasien. In Beckmann M, Perl F: Frauenheilkunde und Geburtshilfe, Basel.

6. ML Gillian, Chaturvedi AK, Lowy DR (2008) HPV prophylactic vaccines and the potential prevention of noncervical cancers in both men and women. Cancer 113(10 Suppl): 3036-3046.

7. Klug SJ, Hukelmann M, Hollwitz B, Duzenli N, Schopp B, et al. (2007) Prevalence of human papillomavirus types in women screened by cytology in Germany. J Med Virol 79(5): 616-625.

8. Cogliano, Baan R, Straif K, Grosse Y, Secretan B, et al. (2005) Carcinogenicity of human papillomaviruses. Lancet Oncol 6(4): 204.

9. Rosenbrock R (2007) HPV-Impfung-Durchbruch der Krebsprävention? Dossier Forum Gesundheitspolitik.

10. European Medicines Agency: Gardasil-European Public Assessment Report.

11. (2008) European Medicines Agency press release on the safety of Gardasil.

12. FDA approval of Gardasil.

13. (2009) FDA Approves New Indication for Gardasil in Prevent Genital Warts in Men and Boys. FDA press, 2526 The HPV vaccination: review and critical review of the two vaccine alternatives.

14. European Medicines Agency: Cervarix-European Public Assessment Report. 
15. Harper MH, Hirsch MS, Mc Govern (2008) BH: Human papillomavirus vaccines. DS Basow (Editor): UpToDate. Waltham, MA.

16. Rambout L, Hopkins L, Hutton B, Fergusson D (2007) Prophylactic vaccination against human papillomavirus infection and disease in women: a systematic review of randomized controlled trials. CMAJ 177(5): 469479.

17. Dam O, Nocon M, Roll S, Vauth C, Willich SN, et al. (2009) Vaccination against human papilloma viruses (HPV) to prevent HPV 16/18 induced cervical carcinomas and their precursors. Series Health Technology Assessment 2627 The HPV vaccination: review and critical review of the two vaccine alternatives $1^{\text {st }}$ edition, 83 .

18. Smith JF, Brownlow M, Brown M, Kowalski R, Esser MT, et al. (2007) Antibodies from women immunized with Gardasil cross-neutralize HPV 45 pseudovirions. Hum Vaccine 3(4): 109-115.

19. Hepburn HM, Kaufmann AM (2009) Nobel Prize for vaccination against cervical cancer. Current data and guidelines. Internist (Berl) 50(5): 617626.

20. Paavonen J, Jenkins D, Bosch FX, Naud P, Salmerón J, et al. (2007) Efficacy of a prophylactic adjuvanvanted bivalent L1 virus like-particle vaccine against infection with human papillomavirus types 16 and 18 in young women: an interim analysis of a phase III double-blind, randomised controlled trial. Lancet 369(9580): 2161-2170.

21. Harper DM (2007) Sustained Immunogenicity and High Efficacy against HPV 16/18 related cervical dysplasia: Long term follow-up through 6.4 years in women vaccinated with Cervarix. Late breaking abstract, I; Society of Gynaecologic Oncologists (SGO) 2008 Annual Meeting on Womens Cancer.

22. Pathirana D, Für das HPV (2008) Management Forum. S3-Leitlinie zur Impfprävention HPV-assoziierter Neoplasien. Chemotherapie Journal.

23. Rambout L, Hopkins L, Hutton B, Fergusson D (2007) Prophylactic vaccination against human papillomavirus infection and disease in women: a systematic review of randomized controlled trials. CMAJ 177(5): 469479.

24. Gall SA, Julio Teixeira, Cosette Wheeler, Paulo Naud, Diane Harper, et al. (2007) Substantial impact on precancerous lesions and HPV infections through 5.5 years in women vaccinated with the HPV-16/18 L1 VLP AS04 candidate vaccine. AACR, 9: 4900.

25. Hildesheim, Herrero R, Wacholder S, Rodriguez AC, Solomon D, et al (2007) Effect of human papillomavirus 16/18 L1 viruslike particle vaccine among young women with preexisting infection: a randomized trial. JAMA 298(7): 743-753.

26. Guilano AR, Palefsky JM, Goldstone S, Moreira ED Jr, Penny ME, et al (2011) Efficacy of Quadrivalent HPV vaccine against HPV infection and Disease in Males. N Engl J Med 364(5): 401-411.

27. Clifford G, Franceschi S, Diaz M, Munoz N, Villa LL (2006) HPV-type-distribution in women withand withou cervical neoplastic diseases. Vaccine. 24(Suppl 3): S3/26-S34.

28. Castellsague X, Diaz M, de Sanjose S (2005) Worldwide human papillomavirus etiology of cervical adenocarcinoa and ist cofactors: implications for screening and prevention. Journal of the National Cancer Institute 97(14): 1066-1071.
29. Munoz N, Bosch FX, Castellsague X, Díaz M, De Sanjose S et al. (2004) Against which human papillomavirus types shall we vaccinate and screen? The international perspective. Int J Cancer 111(2): 278-285.

30. Marra F, Cloutier K, Oteng B, Marra C, Ogilvie G (2009) Effectiveness and cost effectiveness of human papillomavirus vaccine: a systematic review. Pharmacoeconomics 27(2): 127-147.

31. Jit M, Yoon Hong Choi, W John Edmunds (2008) Economic evaluation of human papillomavirus vaccination in the United Kingdom. BMJ 337: a769.

32. Kim JJ, Ortendahl J, Goldie SJ (2009) Cost-Effectiveness of Human Papillomavirus Vaccination and Cervical Cancer Screening in Women older than 30 years in the United States. Annals of Internal Medicine 151(8): 538-545.

33. HPV Market; Human papillomavirus drug market to reach 1.4 billion in 2012, Drug Week. Atlanta 2003: 223.

34. (2008) Prevention of the necessary cervical Ca precursors by vaccination is clearly proven, Interview with Harald zur Hausen. Doctors newspaper.

35. Medical News Today, 5. September 2007.

36. John Stover (1999) The Economic Impact of AIDS in Kenya (S 11). The Policy Project.

37. CDC: Reports of Health Concerns Following HPV-vaccination.

38. (2006) FDA (Food and Drug Administration): Background document for Vaccine and related biological products advisory committee. Gardasil HPV quadrivalent c'vaccine, VRPBAC Meeting S 13.

39. Frankfurter Rundschau (2008) Doubts about effectiveness.

40. uni-bielefeld.de (http://www.uni-ielefeld.de/gesundhw/ag3/downloads.html)

41. Epidemiological Bulletin 32/2009 of 10 August 2009, Robert Koch Institute Berlin.

42. Brotherton JM, Fridman M, May CL, Chappell G, Saville AM, et al. (2011) Early effect of the HPV vaccination programme on cervical abnormalities in Victoria, Australia: an ecological study. Lancet 377(9783): 2085-2092.

43. Gerhardus A (2009) Cervical cancer: How effective is the HPV vaccine? Dtsch Arztebl 106(8): A-330/B-284/C-276.

44. Cylka Menhorn V (2009) HPV vaccination. The study world has been expanded. Dtsch Doctor's sheet 106: A-1185.

45. Pastrana DV, Gambhira R, Buck CB, Pang YY, Thompson CD, et al. (2005) Cross-neutralization of cutaneous and mucosal papillomavirus types with anti-sera to the amino terminus of L2. Virology 337(2): 365-372.

46. Verdecchia A, Francisci S, Brenner H, Gatta G, Micheli A, et al. (2007) Recent Cancer survival in Europe: a 2000-02 period analysis of EUROCARE-4 data. Lancet Oncol 8(9): 784-796.

47. Olsson SE, Villa LL, Costa RL, Petta CA, Andrade RP, et al. (2007) Induction of immune memory following administration of a prophylactic quadrivalent human papillomavirus (HPV) types 6/11/16/18 L1 virus like particle (VLP) vaccine. Vaccine 25(26): 4931-4939. 